\title{
Importance of acute Mycoplasma pneumoniae and Chlamydia pneumoniae infections in children with wheezing
}

\author{
S. Esposito*, F. Blasi**, C. Arosio**, L. Fioravanti*, L. Fagetti**, R. Droghetti*, \\ P. Tarsia***, L. Allegra**, N. Principi*
}

Importance of acute Mycoplasma pneumoniae and Chlamydia pneumoniae infections in children with wheezing. S. Esposito, F. Blasi, C. Arosio, L. Fioravanti, L. Fagetti, R, Droghetti, P. Tarsia, L. Allegra, N. Principi. (C)ERS Journals Ltd 2000.

ABSTRACT: In order to evaluate the role of Mycoplasma pneumoniae and Chlamydia pneumoniae in reactive airway disease, 71 children aged 2-14 yrs with an acute episode of wheezing and 80 age-matched healthy children were studied.

Sera for the determination of specific antibody levels and nasopharyngeal aspirates for the detection of $M$. pneumoniae and $C$. pneumoniae deoxyribonucleic acid were obtained on admission and after 4-6 weeks. All children with wheezing received a standard therapy with inhaled corticosteroids and bronchodilators for 5-7 days; when antibiotic was added on the basis of the judgement of the paediatrician in charge, clarithromycin $15 \mathrm{mg} \cdot \mathrm{kg}^{\text {body }}$ weight $^{-1} \cdot \mathrm{day}^{-1}$ for 10 days was used.

Acute $M$. pneumoniae and $C$. pneumoniae infections were detected significantly more often in children with wheezing than in controls. In patients infected with one of the two pathogens, a history of recurrent wheezing was significantly more frequent than in those without either infection. During a 3-month follow-up period, among nonantibiotic-treated children, those with acute $M$. pneumoniae and/or $C$. pneumoniae infection showed a significantly higher recurrence of wheezing than those without acute $M$. pneumoniae and/or $C$. pneumoniae infection ( $\mathrm{p}=\mathbf{0 . 0 3}$ ).

These results highlight the apparently significant relationship of Mycoplasma pneumoniae and Chlamydia pneumoniae with wheezing in children, particularly in subjects with a history of recurrent episodes, and the possible improvement in the course of reactive airway disease within paediatric patients with acute Mycoplasma pneumoniae and/or Chlamydia pneumoniae infection.

Eur Respir J 2000; 16: 1142-1146.

During the late 1900s, wheezing became one of the most frequent causes of consultation in paediatric practice [1,2]. A number of epidemiological and clinical studies have highlighted most episodes of wheezing occurring in early life as being associated with viral infections, the most frequently encountered agents being respiratory syncytial virus, adenovirus, parainfluenza viruses 1, 2 and 3, influenza virus types $A$ and $B$, and rhinovirus $[3,4]$. The possibility that viruses may interact with the immune and respiratory systems in early life to initiate the complex pathogenetic mechanism leading to asthma has been the matter of considerable study and debate [5]. It is generally recognized that viral respiratory infections often exacerbate established asthma, and there is speculation that they may be associated with the initiation and maintenance of asthma.

Nonviral respiratory pathogens such as Mycoplasma pneumoniae and Chlamydia pneumoniae have also been associated with the possible initiation and promotion of asthma [6-9]. Both M. pneumoniae and C. pneumoniae are the causative agents in a number of respiratory diseases, including upper respiratory tract illnesses such as rhinitis, pharyngitis and otitis, as well as bronchitis and atypical pneumonia [10]. These pathogens are plausible candidates for being aetiological agents in asthma
* Paediatric Dept I, University of Milan, and **Institute of Respiratory Diseases and ***Dept of Emergency Medicine, IRCCS Maggiore Hospital, University of Milan, Milan, Italy.

\author{
Correspondence: N. Princip \\ Paediatric Dept I \\ University of Milan \\ via Commenda 9 \\ 20122 Milan \\ Italy \\ Fax: 39055195341 \\ Keywords: Children \\ Chlamydia pneumoniae \\ Mycoplasma pneumoniae \\ wheezing
}

Received: March 122000

Accepted after revision August 182000 because of their tropism in regard the human respiratory tract and their demonstrated ability to produce chronic respiratory tract infection and inflammation. Further evidence for the role of these pathogens in asthma comes from the observation of improvement in asthma symptoms after antimicrobial therapy active against $M$. pneumoniae and $C$. pneumoniae $[11,12]$.

Most of the published information linking M. pneumoniae or $C$. pneumoniae infection to asthma is derived from studies in adult patients $[6,7,11,12]$. Few data are available regarding childhood $[8,9]$. The aim of the present study was to evaluate the role of $M$. pneumoniae and $C$. pneumoniae in paediatric patients with reactive airway disease.

\section{Subjects and methods}

\section{Study subjects}

Between December 1997 and May 1999, 71 children aged 2-14 yrs, presenting to the Paediatric Emergency Department with an acute episode of wheezing (defined by cough and/or dyspnoea with expiratory rales and wheezes) associated with fever and signs or symptoms of 
upper respiratory tract infection, were studied. During the same time period, 80 healthy subjects of similar sex and age, without any history of respiratory tract infection in the 3 months before enrolment, seen at Paediatric Department for minor surgical problems, were evaluated as control group.

All children with wheezing received a standard therapy with inhaled corticosteroids and bronchodilators for 5-7 days; when antibiotic was added on the basis of the judgement of the paediatrician in charge, clarithromycin 15 $\mathrm{mg} \cdot \mathrm{kg}$ body weight ${ }^{-1} \cdot$ day $^{-1}$ for 10 days was used. The standard treatment with steroids and bronchodilators was equivalent in clarithromycin-treated and nonantibiotictreated children as regards the drugs used and the length of therapy. Clarithromycin was chosen based on the in vitro susceptibility to macrolides of $M$. pneumoniae and $C$. pneumoniae $[13,14]$. The duration of therapy was based on previous clinical observations from use of this antibiotic in children $[15,16]$. Parents and legal guardians were informed of the empirical nature of the therapy. They were told that it represented an unapproved indication for an approved antibiotic likely to be effective against $M$. pneumoniae and $C$. pneumoniae infection, if present. To mitigate expectation bias, they were not informed of the time course of improvement reported by other patients. After admission, children with recurrent or worsening signs and symptoms were asked to return immediately to the study centre for evaluation. The effect of the different therapies on paediatrician-diagnosed wheezing relapses was analysed; positive response to treatment was defined as control of wheezing-related symptoms (i.e. cough, dyspnoea, expiratory rales and wheezes) for the following 3 months.

The study was approved by the Institutional Review Board of the University of Milan, and written informed consent was obtained from the parents or legal guardians of all participants.

\section{Methods}

On admission and after 4-6 weeks, sera for determination of levels of antibodies directed against $M$. pneumoniae and $C$. pneumoniae and nasopharyngeal aspirates for $M$. pneumoniae and $C$. pneumoniae deoxyribonucleic acid (DNA) detection were obtained from all of the participants in the study.

Serum samples were collected and frozen at $-20^{\circ} \mathrm{C}$. Serological studies were performed with an enzyme-linked immunosorbent assay (Pantec, Turin, Italy) for immunoglobulin (Ig) M and IgG directed against M. pneumoniae and a microimmunofluorescence test (Labsystems, Helsinki, Finland) for IgM, IgG and IgA directed against $C$. pneumoniae. Nasopharyngeal aspirate samples in $2 \mathrm{~mL}$ of transport medium containing a sucrose phosphate buffer were frozen at $-70^{\circ} \mathrm{C}$. Nested polymerase chain reaction (PCR) was performed for both pathogens with validated methods, as previously described $[17,18]$. To avoid the risk of contamination, sample preparation, PCR amplification and product analysis were performed in separate rooms. In each assay, positive and negative controls were included. The primer set MP-1 and MP-2 was used for $M$. pneumoniae-specific amplification [17]. The reaction volumes for the first and second rounds of amplification were $50 \mu \mathrm{L}$ with $0.1 \mu \mathrm{M}$ (each) primer. Amplification was carried out for 40 cycles. For M. pneumoniae nested PCR, the primers MUH-1 and MUH-2 were used. Nested amplification was performed, using $5 \mu \mathrm{L}$ 1:10-diluted PCR product ( $5 \mu \mathrm{L}$ in $45 \mu \mathrm{L}$ sterile water) from the first round of amplification under identical conditions. Touchdown nested PCR for detection of $C$. pneumoniae DNA was performed using primers designed to detect the major outer membrane protein [19]. Extracted DNA solution $(10 \mu \mathrm{L}$ in a total volume of $50 \mu \mathrm{L})$ was used in the first PCR round; $5 \mu \mathrm{L}$ of the PCR products amplified by the outer primers was then transferred to a new $50-\mu \mathrm{L}$ PCR reaction mix for a second amplification using the inner primers [18]. The first round consisted of 40 cycles and the second 35 .

Acute $M$. pneumoniae and/or C. pneumoniae infection was diagnosed if the patient showed a significant antibody response to one of the pathogens in paired sera $(M$. pneumoniae: specific $\operatorname{IgM} \geq 1: 100$, specific $\operatorname{IgG} \geq 1: 400$, or a four-fold increase in IgG titre; C. pneumoniae: specific $\operatorname{IgM} \geq 1: 16$, specific $\operatorname{IgG} \geq 1: 512$, or a four-fold increase in IgG titre) and/or if the PCR on nasopharyngeal aspirates was positive for DNA from one of the two organisms [20].

In children with wheezing and in controls, skin-prick tests for common allergens were performed on the arm to demonstrate allergen sensitization [21]. Children were to have refrained from taking antihistamines or antidepressants for $\geq 72 \mathrm{~h}$ and from topical or systemic corticosteroids for $\geq 7$ days. Both positive $\left(10 \mathrm{mg} \cdot \mathrm{mL}^{-1}\right.$ histamine) and negative (saline/glycerol 50/50) controls were included. A standard battery of extracts were tested. These were all Soluprick SQ 10 HEP (ALK A/S, Hörsholm, Denmark): hen's egg white, cow's milk, house dust mite (Dermatophagoides pteronyssinus and $D$. farinae), dog and cat dander, birch and timothy grass pollen, mugwort (Artemisia vulgaris), Aspergillus mix and Alternaria tenuis. The size of the reactions was measured 15 min after testing. The histamine weal size was recorded as the sum of the longest plus the midpoint orthogonal diameters divided by 2. A child was considered atopic to a specific allergen if the mean diameter of the weal was at least half that produced by a $10-\mathrm{mg} \cdot \mathrm{mL}^{-1}$ solution of histamine, and with a mean diameter of the weal due to histamine of $\geq 3 \mathrm{~mm}$ [21]. Atopy was defined by at least one positive skin-prick test.

\section{Statistical analysis}

Comparisons between the groups were performed using Fisher's exact test or the Chi-squared test. Age comparison was performed using an unpaired t-test. A p-value of $<0.05$ was considered significant.

\section{Results}

\section{Study subjects}

Table 1 shows the demographic characteristics of the study population. No significant difference was observed in sex, age, prevalence of atopy or eczema, and family history of atopic diseases and asthma between children with wheezing and controls. Among children with wheezing, $31(43.7 \%)$ had a history of paediatrician-diagnosed recurrent episodes (i.e. at least four acute episodes of wheezing in the 12 months preceding enrolment), 
Table 1. - Demographic characteristics of the study population

\begin{tabular}{lcc}
\hline & $\begin{array}{c}\text { Children with } \\
\text { wheezing }\end{array}$ & Controls \\
\hline Subjects n & 71 & 80 \\
Males & $32(45.1)$ & $38(47.5)$ \\
Age yrs & $4.5(2-14)$ & $5.4(2-14)$ \\
Recurrent episodes of wheezing & $31(43.7)^{+}$ & 0 \\
Atopy & $22(30.9)$ & $14(17.5)$ \\
Eczema & $7(9.8)$ & $3(3.7)$ \\
Family history of atopic diseases & $11(15.4)$ & $6(7.5)$ \\
Family history of asthma & $7(9.8)$ & $4(5.0)$ \\
\hline
\end{tabular}

Data are presented as absolute numbers with percentages in parentheses or median (range). ${ }^{+}: \mathrm{p}<0.0001$ versus controls (Chi-squared test).

whereas it was the first episode of wheezing for 40 $(56.3 \%)$ children. None of the controls showed a history of recurrent episodes of wheezing.

Incidence of acute Mycoplasma pneumoniae and Chlamydia pneumoniae infection

Acute $M$. pneumoniae infection was demonstrated in 16 of the $71(22.5 \%)$ children with wheezing: it was serologically determined in all 16 infected patients (specific $\operatorname{IgM} \geq 1: 100$ in 12 children and IgG titre $\geq 1: 400$ in four), and confirmed by PCR in one subject (who presented with an IgG titre of $\geq 1: 400$ ); in none of the patients was $M$. pneumoniae DNA detected without any evidence of seroconversion. Among the controls, six of the 80 (7.5\%) children showed evidence of acute $M$. pneumoniae infection without any respiratory symptom (children with wheezing versus controls, $\mathrm{p}=0.01$ (Chi-squared test): all of the six subjects presented serological evidence of acute infection (specific IgM $\geq 1: 100$ in two children and $\mathrm{IgG}$ titre $\geq 1: 400$ in four); in none of the controls was $M$. pneumoniae DNA detected.

Acute $C$. pneumoniae infection was shown in 11 of the $71(15.5 \%)$ patients with wheezing: it was serologically determined in nine of the 11 infected children (specific IgG $\geq 1: 512$ in one child and a four-fold rise in IgG titre in eight), and confirmed by PCR in four of the nine (in the child with specific $\operatorname{IgG} \geq 1: 512$ and in three of those with a four-fold rise in IgG litre); in two further patients, $C$. pneumoniae DNA was detected without any evidence of seroconversion. Among the controls, two of the 80 (2.5\%) children showed evidence of acute $C$. pneumoniae infection without any respiratory symptom (children with

Table 2. - Incidence of acute Mycoplasma pneumoniae and Chlamydia pneumoniae infection in the study population in different age groups

\begin{tabular}{lrll}
\hline & $\begin{array}{c}\text { Children with } \\
\text { wheezing }\end{array}$ & Controls & p-value \\
\hline $\begin{array}{l}\text { M. pneumoniae } \\
\text { 2-4 yrs }\end{array}$ & $4 / 40(10.0)$ & $2 / 34(5.9)$ & $0.68^{*}$ \\
Z5 yrs & $12 / 31(38.7)$ & $4 / 46(8.7)$ & $0.0003^{+}$ \\
C. pneumoniae & & & \\
2-4 yrs & $4 / 40(10.0)$ & $2 / 34(5.9)$ & $0.68^{*}$ \\
$\geq 5$ yrs & $7 / 31(22.6)$ & $0 / 46(0)$ & $0.001^{+}$ \\
\hline
\end{tabular}

Data are presented as absolute values with percentages in parentheses. *: Fisher's exact test; ${ }^{+}$: Chi-squared test.
Table 3. - Incidence of recurrent episodes in children with wheezing in different age groups

\begin{tabular}{lrrr}
\hline $\begin{array}{l}\text { Age group } \\
\text { yrs }\end{array}$ & Infected $^{\#}$ & Not infected & p-value \\
\hline $2-4$ yrs & $8 / 8(100.0)$ & $9 / 32(28.1)$ & $0.003^{*}$ \\
$\geq 5$ yrs & $12 / 16(75.0)$ & $2 / 15(13.3)$ & $0.002^{+}$ \\
\hline
\end{tabular}

Data are presented as absolute numbers with percentages in parentheses. ": with Mycoplasma pneumoniae and/or Chlamydia pneumoniae; *: Fisher's exact test; ${ }^{+}$: Chi-squared test.

wheezing versus controls, $\mathrm{p}=0.01$ (Chi-squared test)): both subjects showed C. pneumoniae DNA without seroconversion.

Among the infected children, three patients with wheezing and none of the controls showed M. pneumoniae and $C$. pneumoniae coinfection.

Considering studies that have shown the highest incidence of atypical bacterial infections in children of $>5$ yrs [16, 22], a sub-analysis was performed, comparing subjects with wheezing and controls aged 2-4 yrs and those of $>5$ yrs. Table 2 summarizes the incidence of acute $M$. pneumoniae and $C$. pneumoniae infection in the study population in different age groups. Despite the higher incidence of infection due to either pathogen in children with wheezing than in controls in both age groups, the difference was significant only in subjects aged $>5$ yrs.

Fifteen of the $16(93.7 \%)$ wheezing patients with acute $M$. pneumoniae infection had a history of recurrent wheezing, as against only 16 of the $55(29.1 \%)$ without $(\mathrm{p}<0.0001$ (Chi-squared test)); results were similar for acute $C$. pneumoniae infection: eight of $11(72.7 \%)$ as against 23 of $60(38.3 \%)(\mathrm{p}=0.04$ (Chi-squared test)).

Table 3 details the incidence of recurrent episodes in children with wheezing in different age groups. In both age groups, a history of recurrent wheezing was significantly more frequent in the patients infected by one of the two pathogens than in those without either infection.

No significant difference in the prevalence of atopy was found between the wheezing subjects with and without infections due to these pathogens. In the group of 16 patients with wheezing and acute $M$. pneumoniae infection, six (37.5\%) appeared to be atopic, whereas, among the 55 with wheezing but no $M$. pneumoniae infection, $16(29.1 \%)$ were atopic ( $\mathrm{p}=0.54$ (Fisher's exact test)). Similarly, among the 11 patients with wheezing and acute $C$. pneumoniae infection, four $(36.7 \%)$ were atopic, whereas, among the 60 with no C. pneumoniae infection, $18(30.0 \%)$ subjects showed evidence of atopy $(\mathrm{p}=0.72$ (Fisher's exact test)).

\section{Antibiotic treatment}

Twenty-two of the $71(30.9 \%)$ children with wheezing, in addition to standard therapy with steroids and bronchodilators, received clarithromycin, irrespective of serological and PCR results. Among the antibiotic-treated patients, $11(50.0 \%)$ showed evidence of acute $M$. pneumoniae and/or C. pneumoniae infection. In 13 of the 49 (26.5\%) nonantibiotic-treated subjects, evidence of acute $M$. pneumoniae and/or $C$. pneumoniae infection was found. 
Table 4. - Clinical outcome of wheezing in nonantibiotictreated children during the 3-month follow-up, according to diagnosis of infection

\begin{tabular}{lcc}
\hline & Infected* & Not infected* \\
\hline Clinical resolution & $4(30.8)$ & $25(69.4)^{+}$ \\
Recurrence & $9(69.2)$ & $11(30.6)^{+}$ \\
\hline
\end{tabular}

Data are presented as absolute numbers with percentages in parentheses. *: with Mycoplasma pneumoniae and/or Chlamydia pneumoniae. ${ }^{+}: \mathrm{p}=0.03$ (Chi-squared test).

During the 3-month follow-up period, among children with evidence of acute $M$. pneumoniae and/or C. pneumoniae infection, nine of the $13(69.2 \%)$ nonantibiotic-treated subjects showed recurrence of wheezing; conversely, none of the clarithromycin-treated patients showed a new episode of wheezing ( $\mathrm{p}=0.0005$, (Fisher's exact test)).

Table 4 summarizes the clinical outcome of wheezing in nonantibiotic-treated children during the 3-month follow-up, according to diagnosis of infection. Significantly more recurrence of wheezing was found in children with acute $M$. pneumoniae and/or $C$. pneumoniae infection than in those without acute $M$. pneumoniae and/or $C$. pneumoniae infection ( $\mathrm{p}=0.03$ (Chi-squared test)).

Follow-up PCR at 4-6 weeks in patients for whom it was positive on admission was negative for $M$. pneumoniae DNA in one antibiotic-treated child, whereas it was still positive for $C$. pneumoniae DNA in two of three clarithromycin-treated children.

\section{Discussion}

The present study indicates that $M$. pneumoniae and $C$. pneumoniae are significantly related to wheezing in children, particularly in subjects with a history of recurrent episodes, and that clarithromycin therapy may improve the course of reactive airway disease in paediatric patients with acute $M$. pneumoniae and/or $C$. pneumoniae infection.

The finding of a relationship between wheezing episodes and acute $M$. pneumoniae or $C$. pneumoniae infection is intriguing and suggests a potential role for these pathogens in the exacerbation of childhood asthma. It is likely that $M$. pneumoniae and $C$. pneumoniae can trigger the "wheezing process" in subjects who are predisposed by either their genetic background or events that have "primed" their immune systems and lungs.

In agreement with previous reports, the present results also show that, in children with wheezing, the incidence of acute $M$. pneumoniae and $C$. pneumoniae infection increases with age and occurs mainly after 5 yrs of age $[8$, 9]. Moreover, in the present study population, the incidence of asymptomatic infection in healthy subjects seemed to be low and was similar to that recently reported in adults $[23,24]$.

The present data support the concept that measurement of the antibody response in paired sera represents an accurate diagnostic test for determining the aetiology of acute $M$. pneumoniae or $C$. pneumoniae infection. In the present study, there was a relationship between serological evidence of acute infection with the two pathogens and wheezing status. All children with acute $M$. pneumoniae infection met accepted serological criteria, and in none of them was $M$. pneumoniae DNA detected without any evidence of seroconversion. Only in two children with wheezing and two controls was $C$. pneumoniae DNA detected without any evidence of seroconversion. However, results with PCR positive for C. pneumoniae in the absence of diagnostic antibody have been reported in paediatric age by other authors and may be caused by an immature ability to produce a humoral response or poor antigenic stimulation after $C$. pneumoniae infection [22].

Transient elevation of total serum IgE levels has been demonstrated during the acute phase of viral as well as $M$. pneumoniae infections, even in the absence of wheezing [25]. Moreover, EMRE et al. [26] demonstrated the presence of anti- $C$. pneumoniae IgE by immunoblotting in $85.7 \%$ of culture-positive children with wheezing, in contrast to only $9.1 \%$ of culture-positive patients with community-acquired pneumonia who were not wheezing. Thus the IgE response seems to be an integral part of the host response to a variety of infections.

The present report highlights the link between wheezing, atopy defined by positive skin-prick tests and atypical bacterial infections. Interestingly, no significant difference in the prevalence of atopy was found between wheezing children with and without infections due to either pathogen. However, further studies of the relationship between M. pneumoniae and C. pneumoniae infection and atopy are needed to provide a more comprehensive understanding of how these triggers for wheezing interact.

Regarding antimicrobial therapy, macrolides are the only drugs active against $M$. pneumoniae and $C$. pneumoniae that can be safely used when treating paediatric patients $[15,16]$. Previous studies have demonstrated the efficacy of macrolides in the treatment of atypical bacterial infections in children with community-acquired pneumonia $[15,16]$. EMrE et al. [8] observed that nine of 12 children with asthma and positive cultures for $C$. pneumoniae demonstrated clinical and laboratory improvement of their symptoms following antibiotic therapy with clarithromycin for 10 days or erythromycin for 14 days. However, in children with wheezing, the efficacy of antibiotic treatment and the optimal length of therapy have not been established. Furthermore, it is well known that macrolides have anti-inflammatory activities due to an interaction with the natural effectors involved in antimicrobial defences and inflammation [27]. This interaction has been shown to affect bronchial hyperresponsiveness and to improve clinical status in patients with asthma, but the importance of this property has not been fully elucidated [28].

In the present study population, clarithromycin, 15 $\mathrm{mg} \cdot \mathrm{kg}$ body weight ${ }^{-1}$. day ${ }^{-1}$ for 10 days, seemed to be effective in the resolution of wheezing-related symptoms in subjects with atypical bacterial infections. Respiratory symptoms frequently recur in the absence of antimicrobial treatment of acute $M$. pneumoniae and/or C. pneumoniae infection. On the contrary, all 11 patients with acute $M$. pneumoniae and/or C. pneumoniae infection treated with clarithromycin appeared to benefit from the macrolide even though, in some patients, PCR results remained still positive. Considering that therapy with inhaled steroids was equivalent in both groups with regard to drugs used and the length of treatment, this benefit seems to be related to clarithromycin's activity. Preliminary experience in adults suggests that $M$. pneumoniae and $C$. pneumoniae may prove difficult to eradicate with the currently available antibiotic regimens [29]. HAMMERSCHLAG et al. [29] described 
a number of subjects who remained culture-positive for C. pneumoniae despite treatment with one or more courses of tetracycline or doxycycline of up to 3 weeks' duration. Even when a subject becomes culture-negative, this does not necessarily mean that the organism has been eradicated; it may persist in the body in a latent form [30].

A limitation of the present report is the lack of respiratory virus testing. It is possible that $M$. pneumoniae and $C$. pneumoniae infections act as cofactors, possibly rendering subjects more susceptible to other stimuli such as viruses.

In conclusion, the present preliminary results show a possible association of infection with Mycoplasma pneumoniae and Chlamydia pneumoniae and wheezing, particularly in children with recurrent episodes. Clarithromycin-treatment of paediatric patients with wheezing and atypical bacterial infection appears to be associated with clinical improvement. Further studies are needed to clarify the actions of macrolides in subjects with asthmalike manifestations. However, in children whose wheezing-related symptoms remain poorly controlled, a careful search for evidence of Mycoplasma pneumoniae and Chlamydia pneumoniae infection may be indicated. The present results also support the argument that definitive randomized double-blind trials of antibiotics in various wheezing children populations should be carried out, since, if these preliminary findings can be confirmed, the benefit to patients could be significant.

Acknowledgements. The authors would like to thank C. Cantoni, C. Dotti and V. Popescu Janu for their excellent technical assistance.

\section{References}

1. Milgrom H, Wood II RP, Ingram D. Respiratory conditions that mimic asthma. Immunol Allergy Clin North Am 1998; 18: 113-132.

2. Martinez FD, Helms PJ. Types of asthma and wheezing. Eur Respir J 1998; 12: Suppl. 27, 3S-8S.

3. Duff AL, Pomeranz ES, Gelber LE, et al. Risk factors for acute wheezing in infants and children: viruses, passive smoke, and IgE antibodies to inhalant allergens. Pediatrics 1993; 92: 535-540.

4. Newson R, Strachan D, Archibald E, Emberlin J, Hardaker P, Collier C. Acute asthma epidemics, weather and pollen in England, 1987-1994. Eur Respir J 1998; 11: 694-701.

5. Martinez FD. Viral infections and the development of asthma. Am J Respir Crit Care Med 1995; 151: 1644-1648.

6. Kraft M, Cassell GH, Henson JE, et al. Detection of Mycoplasma pneumoniae in the airways of adults with chronic asthma. Am J Respir Crit Care Med 1998; 158: 998-1001.

7. Hahn DL, Dodge RW, Goulgjatnikov R. Association of Chlamydia pneumoniae (strain TWAR) infection with wheezing, asthmatic bronchitis, and adult-onset asthma. JAMA 1991; 266: 225-230.

8. Emre U, Roblin PM, Gelling M, et al. The association of Chlamydia pneumoniae infection and reactive airway disease in children. Arch Pediatr Adolesc Med 1994; 148: 727-732.

9. Cunningham AF, Johnston SL, Julious SA, Lampe FC, Ward ME. Chronic Chlamydia pneumoniae infection and asthma exacerbations in children. Eur Respir J 1998; 11: 345-349.

10. File TM, Tan JS, Plouffe JF. The role of atypical patho- gens: Mycoplasma pneumoniae, Chlamydia pneumoniae, and Legionella pneumophila in respiratory infection. Infect Dis Clin North Am 1998; 12: 569-592.

11. Hahn DL. Treatment of Chlamydia pneumoniae infection in adult asthma: a before-after trial. J Fam Practice 1995; 41: 345-351.

12. Black PN, Bagg B, Brodie SM, Robinson E, Cooper B. A double-blind, crossover study of roxithromycin in the treatment of asthma. Eur Respir J 1998; 12: Suppl. 28, 190S.

13. Taylor-Robinson D, Bebear C. Antibiotic susceptibilities of mycoplasmas and treatment of mycoplasmal infections. J Antimicrob Chemother 1997; 40: 622-630.

14. Roblin PM, Montalban G, Hammerschlag MR. Susceptibilities to clarithromycin and erythromycin of isolates of Chlamydia pneumoniae from children with pneumonia. Antimicrob Agents Chemother 1994; 38: 1588-1589.

15. Harris JS, Kolokathis A, Campbell M, Cassell G, Hammerschlag M. Safety and efficacy of azithromycin in the treatment of community-acquired pneumonia in children. Pediatr Infect Dis J 1998; 17: 865-871.

16. Block S, Hedrick J, Hammerschlag MR, Cassell GH, Craft JC. Mycoplasma pneumoniae and Chlamydia pneumoniae in pediatric community-acquired pneumonia: comparative efficacy and safety of clarithromycin $v$ s. erythromycin ethylsuccinate. Pediatr Infect Dis J 1995; 14: 471-477.

17. Abele-Horn M, Busch U, Nitzschiko H, et al. Molecular approaches to diagnosis of pulmonary diseases due to $\mathrm{Myco-}$ plasma pneumoniae. J Clin Microbiol 1998; 36: 548-551.

18. Blasi F, Boman J, Esposito G, et al. Chlamydia pneumoniae DNA detection in peripheral blood mononuclear cells is predictive of vascular infection. J Infect Dis 1999; 180: 2074-2076.

19. Tong CYW, Sillis M. Detection of Chlamydia pneumoniae and Chlamydia psittaci in sputum samples by PCR. J Clin Pathol 1993; 46: 313-317.

20. Wubbel L, Muniz L, Ahmed A, et al. Etiology and treatment of community-acquired pneumonia in ambulatory children. Pediatr Infect Dis $J$ 1999; 18: 98-104.

21. Nystad W, Skrondal A, NjA F, Hetlevik Ø, Carlsen KH, Magnus P. Recurrent respiratory tract infections during the first 3 years of life and atopy at school age. Allergy 1998; 53: 1189-1194.

22. Normann E, Gnarpe J, Gnarpe H, Wettergren B. Chlamydia pneumoniae in children with acute respiratory tract infections. Acta Paediatr 1998; 87: 23-27.

23. Gnarpe J, Lundback A, Sundelof B, Gnarpe H. Prevalence of Mycoplasma pneumoniae in subjectively healthy individuals. Scand J Infect Dis 1992; 24: 161-164.

24. Gnarpe J, Gnarpe H, Sundelof B. Endemic prevalence of Chlamydia pneumoniae in subjectively healthy persons. Scand J Infect Dis 1991; 23: 387-388.

25. Nagayama Y, Sakurai N. Clinical observations on lower respiratory tract infections with special reference to serum IgE levels. Pediatr Pulmonol 1991; 11: 44-48.

26. Emre U, Sokolovskaya N, Roblin PM, Schachter J, Hammerschlag M. Detection of anti-Chlamydia pneumoniae $\operatorname{IgE}$ in children with reactive airway disease. $J$ Infect Dis 1995; 172: 265-267.

27. Labro MT. Anti-inflammatory activity of macrolides: a new therapeutic potential? J Antimicrob Chemother 1998; 41: Suppl. B, 37-46.

28. Wales D, Woodhead M. The anti-inflammatory effects of macrolides. Thorax 1999; 54: Suppl. 2, 558-562.

29. Hammerschlag MR, Chirgwin K, Roblin PM, et al. Persistent infection with Chlamydia pneumoniae following acute respiratory illness. Clin Infect Dis 1992; 14: 178-182.

30. Black PN. The use of macrolides in the treatment of asthma. Eur Respir Rev 1996; 6: 240-243. 